\title{
A dual-loop control system of grasping force for prosthetic hands
}

\author{
Congbo $\mathrm{Li}^{1,2}$, Xiaobao Deng ${ }^{1,2}$ and Qunming $\mathrm{Li}^{1,2, \mathrm{a}}$ \\ ${ }^{1}$ State key laboratory of High-Performance Complex Manufacturing \\ ${ }^{2}$ School of Mechanical \& Electrical Engineering, Central South University, Changsha, 410083, China
}

\begin{abstract}
Despite the rapid developments of prosthetic hands technologies in recent years, there still exists a huge gap between prosthetic hands and human hands in flexibility. The limitation of sensors attached to the prosthetic hand is one of the causes, and another reason is that the materials, weight, and stiffness of the object gripped by a prosthetic hand are unknown. Additionally, the external disturbances in prosthetic hands are not certain. The factors mentioned above lead to difficulties in controlling grasping force of prosthetic hands, and that affects the quality of prosthetic hands applications. Therefore, it is important to design a stable and reliable system to control grasping force. This paper presents a dual-loop control system of grasping force for prosthetic hands, and we carried out some relative experiments based on a single degrees of freedom prosthetic hand. The experiment results show that the proposed control system is effective.
\end{abstract}

Keywords: dual-loop control system; prosthetic hands; reflex control; grasping force; fuzzy controller.

\section{Introduction}

Hands are an essential part of a human body, but some people may lose their hands due to some incidents, such as diseases, work injuries, traffic incidents, etc. Those people face big challenges in their daily life, and someone even has serious mental illnesses. To solve these problems, the prosthetic hand has been created. There are different types of prosthetic hands. According to the degrees of freedom (DOF), prosthetic hands are classified into single DOF hands and multi-DOF hands; according to the ways of actuation, prosthetic hands can be classified into hydraulic-drive hands, electric-drive hands, etc. During its evolution, the prosthetic hand has experienced changes from bigsized, two-finger to small-sized, multi-fingered. Meanwhile, the prosthetic hand turns preciselygrasped and becomes more functional compared with its initial functions like simple grasp. Generally speaking, tendencies of prosthetic hands' developments are humanoid, multi-functional and highly integrated. The ultimate goal of the invention of prosthetic hands is to offer people who lose hands a normal life.

The appearance of the prosthetic hand brings hope to the disabled, but some problems arose which restrict its further developments. For getting a better and functional prosthetic hand, designing a stable and reliable control system is a key point. As demonstrated in [1-3], methods for controlling prosthetic

\footnotetext{
a Corresponding author : liqm@mail.csu.edu.cn
} 
hands are proposed. Even if a prosthetic hand is shaped exactly like humans' hands, it can't act well without a good control system. So it is an urgency to improve the control system. On the basis of researches conducted in recent years, the control system of prosthetic hands includes open-loop control systems and close-loop control systems. The biology-decoding technology has been applied to the open-loop control, enabling people to control prosthetic hands directly. The close-loop system like sliding mode control [4], PID control [5] and EMG proportional control [6] is widely used. Both the open-loop systems and close-loop systems have advantages and disadvantages, and they all play an indispensable role in the control of prosthetic hands. Nevertheless, the close-loop systems mentioned in the following contents are mainly applied in prosthetic hands.

\section{Problem formulation}

Nowadays using the biologic signals of human bodies to control prosthetic hands directly is a major method in an open-loop control system. By decoding the EMG signals, EEG signals and peripheral nerve signals, the system can obtain the human intentions from brains, and sends out instructions which are scaled up to control the prosthetic hand. When the grasping is not stable, people who wear a prosthetic hand can adjust the intensity of biologic signals to control the grasping force with visual feedbacks. However, there are many difficulties in the biologic-decoding control system. For instance, the biologic signals acquired from sensors are weak, and with the time passing, some drifts of those signals may happen, which generates some interference in controlling processes. Furthermore, it takes a long time to decode the biologic signals, resulting in some delays in controlling prosthetic hands. In that case, the real-time control is hard to realize. Generally, applying the biologic-decoding method to gain information about shapes, sizes or weight of objects gripped by prosthetic hands is limited. Only some simple grasping modes can be realized in an open-loop system.

The closed-loop control is the key to promoting applications of the prosthetic hand, which is also important to realize an efficient and stable grasp. Many researchers have proposed a kind of closedloop control system of grasping force based on position sensors and tactile sensors. Control strategies used in this system are mostly the impedance control. It could adjust the grasping force by using the feedback information to control the position of hands. Some methods about grasping force control proposed in [7-8] is efficient in controlling the grasping force of prosthetic hands. For now, how to design a closed-loop system to control the grasping force of prosthetic hands well is an urgent issue, and it is a significant step to promote the marketization of prosthetic hands.

\section{The structure of dual-loop control system}

Researches have shown that when people are ready to grasp an object, they will observe the features of this object firstly, and then the nerve system conveys the feature information to the brain. Next, the brain estimates a proper grasping force according to life experience, and sends a grasping order to the body. As soon as the skeletal muscles get a grasping order from the nerve system, they will shrink to make the grasp happen. If the force is too small, people's tactile and slip systems will feed back the signal to the spinal cord nerve system. With these feedbacks, human hands will increase grasping force by shrinking muscles to make sure the object gripped will not slid.

When grasping an object, human hands can adjust the force at any moment, making sure the object will not become deformed or slid. After comparing with the grasping strategies of human hands, we propose a dual-loop control system based on DSP28335 to control the grasping force.

The dual-loop control system consists of the controlling and driving circuitry, the DSP28335 controller, sensors, etc. The core of the dual-loop system is a DSP28335 controller. It includes hardware and software, and the hardware is shown in Figure 1. Also, the programming of software is accomplished in CCS 3.3 provided by Texas Instruments firm. 


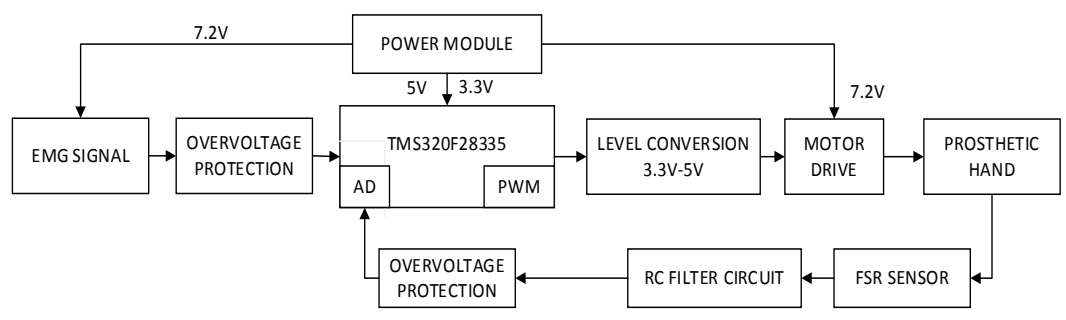

Figure 1. Hardware of dual-loop system

The dual-loop system has two modes used in grasping force control. One is called the primary control, and the other is called the reflex control. In the primary control, a rule base of fuzzy controller based on FSR is built to make the grasp real-time. The structure of the fuzzy controller is shown in Figure 2.

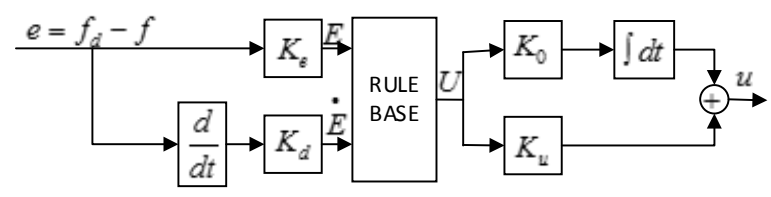

Figure 2. Structure of fuzzy control

Besides, $f_{d}$ is the expected grasping force, and $f$ is the real grasping force. $K_{e}, K_{d}, K_{0}$ and $K_{u}$ are the ratio coefficients. The FSR, whose functions are introduced in [9-11], is in charge of detecting grasping force and generating relative signals. With the grasping force signals gained at the first touch, the primary control system evaluates an expected force and gives instructions to the fuzzy controller, which is widely used for industrial process [12-15]. Then the fuzzy controller can control grasping force to make the grasp more precise. The primary control aims to simulate the human grasping process, which is a fuzzy control process, so we use the fuzzy controller to realize it. To test the primary control, the fuzzy controller is used in experiments in comparison with the digit PID controller, whose mathematical expressions can be described as:

$$
\begin{aligned}
& u(k)=u(k-1)+q_{0} e(k)+q_{1} e(k-1)+q_{2} e(k-2) \\
& q_{0}=K_{p}\left(1+\frac{T_{d}}{T_{0}}\right), q_{1}=-K_{p}\left(1+2 \frac{T_{d}}{T_{0}}-\frac{T_{0}}{T_{i}}\right), q_{2}=K_{p} \frac{T_{d}}{T_{0}}
\end{aligned}
$$

where $T_{0}$ is the sample period, $T_{i}$ is the integration constant, $T_{d}$ is the differential constant and $K_{p}$ is the proportional gain.

In the reflex control, when disturbances happen in the grasping period, the grasping force is adjusted by the control system to avoid sliding or destruction of objects. Two modes' block diagram in the dual-loop control system is showed in Figure 3 and Figure 4.

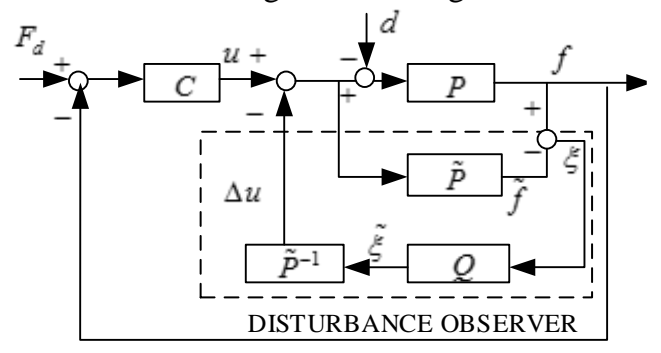

Figure 3. The reflex control 


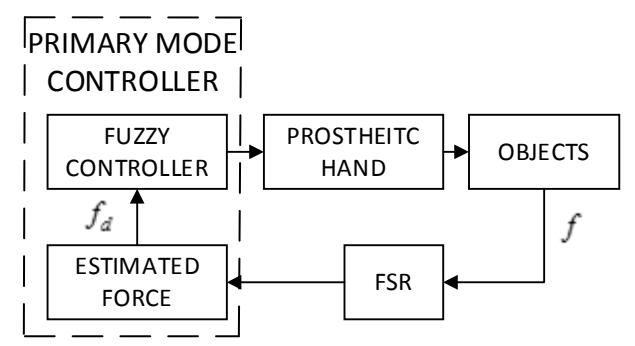

Figure 4. The primary control

\section{Experiments and results}

To test the dual-loop system's abilities in controlling grasping force, we conducted some grasping experiments based on the primary control, the reflex control and the dual-loop control with a single degree of freedom prosthetic hand, whose transfer function can be described as follows:

$$
G(s)=\frac{1.4667}{0.0047 s^{2}+0.1565 s+1}
$$

The core of our experimental platform is the F28335 chip made by TI firm, and the motors are controlled by the PWM technology. Our whole experimental platform is shown in Figure 5.

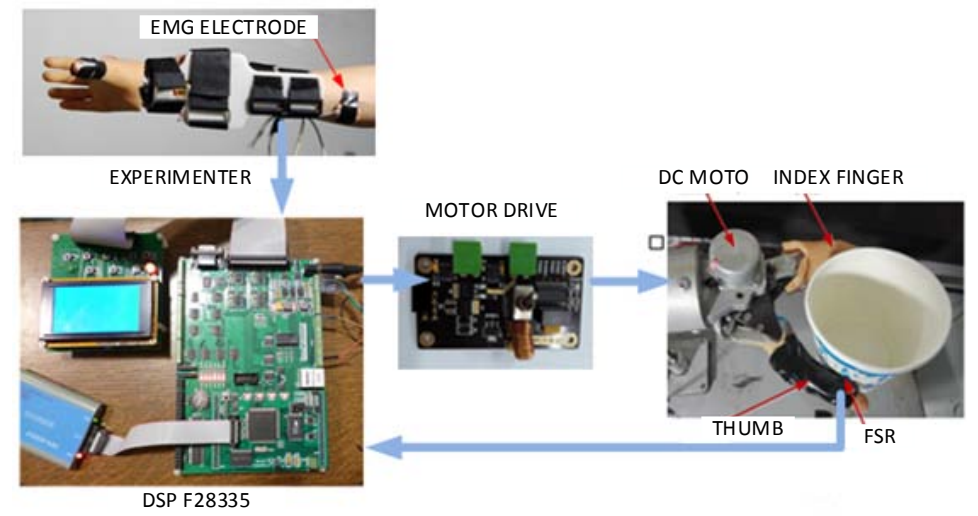

Figure 5. Experimental platform

\subsection{The primary control experiment}

Three objects with different hardness are used in this grasping experiment. We applied the digital PID control algorithm and the fuzzy control algorithm to make a comparison. By the test-and-error method, the parameters of PID and Fuzzy algorithms can be acquired as follows in Table 1. The sample rate used in this experiment is $1000 \mathrm{~Hz}$.

Table 1. Parameters of the controller

\begin{tabular}{cccccccc}
\hline Parameter & $q_{0}$ & $q_{1}$ & $q_{2}$ & $K_{e}$ & $K_{d}$ & $K_{0}$ & $K_{1}$ \\
Value & 9 & 0.08 & 3 & 0.5 & 0.2 & 0.001 & 0.2 \\
\hline
\end{tabular}

Results of grasping the hard object are as below: 

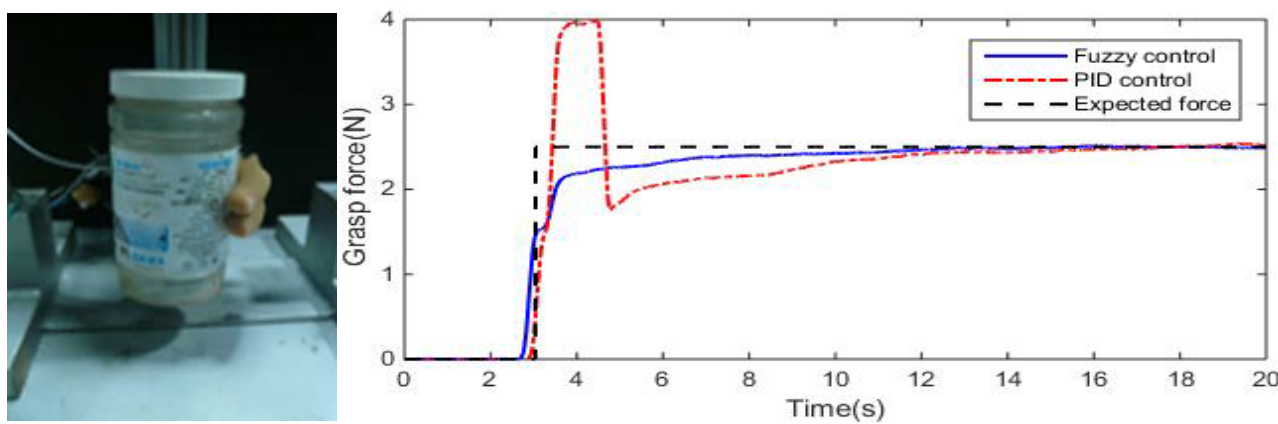

Figure 6. Date of grasping a glass bottle

Results of grasping the middle-hardness object are shown as below:
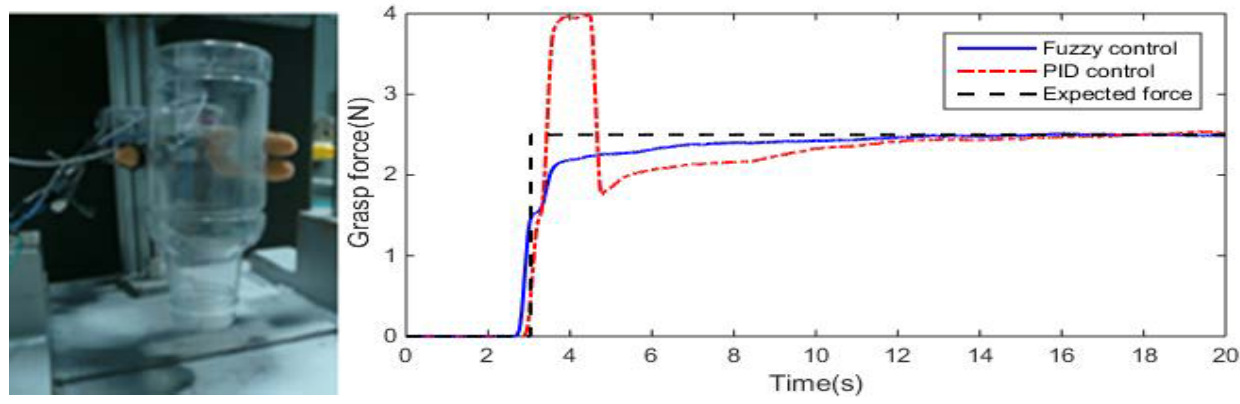

Figure 7. Date of grasping a plastic bottle

Results of grasping the soft object are as below:
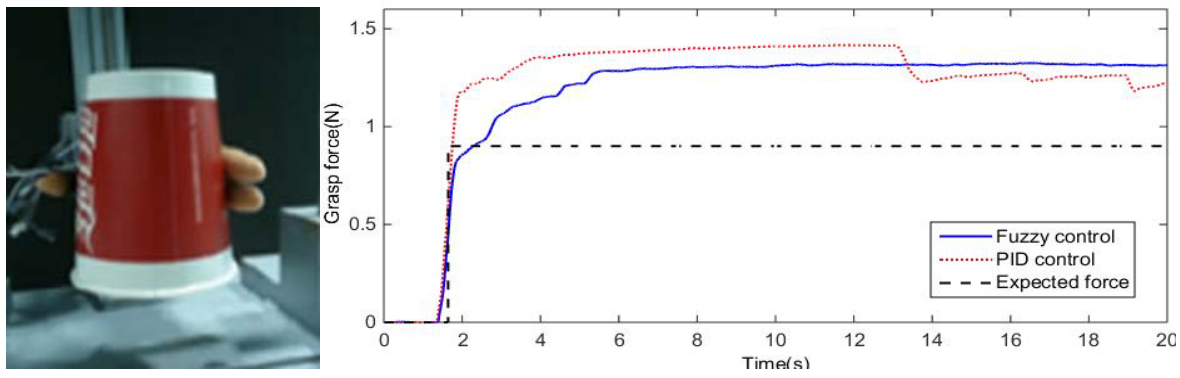

Figure 8. Date of grasping a paper cup

The materials of grasped objects, the expected grasp force, weight of objects, driving voltage and initial voltage of the primary control mode experiment are showed in Table 2.

Table 2. Experiment Parameters of the primary control mode

\begin{tabular}{ccccc}
\hline MATERIAL & WEIGHT(g) & EXPECTED & DRIVING & INITIAL \\
GLASS & 413 & 2.5 & VOLTAGE(V) & VOLTAGE(V) \\
PLASTIC & 30 & 1.5 & 1.5 & 1 \\
PAPER & 12 & 0.9 & 1.5 & 1 \\
\hline
\end{tabular}

From these experiment results, it is convinced that the fuzzy control algorithm, which is used in the primary control, can give a more stable grasping force compared with the digital PID control algorithm. By the way, the overshooting is less when we use the fuzzy algorithm. In a word, the primary control system enables the prosthetic hand to grasp an object stably without destruction. 


\subsection{The reflex control experiment}

The reflex experiment based on the disturbance observer was conducted with three objects of different hardness, and the fuzzy control algorithm was used. The disturbing device is shown in Figure 9. The FSR sensor is stuck in the thumb to measure grasping force signals. The DSP control system takes charge of making prosthetic hand move, and the pressure bar of manometer hits the object griped by prosthetic hands with a certain amount of pressure, which could make the object slide. Using the manometer to hit the prosthetic hand when a hard object is grasped stably, and results are shown in Figure 10. The result of hitting a middle-hardness object is shown in Figure 11. The results of hitting a middle-hardness object are shown in Figure 12.

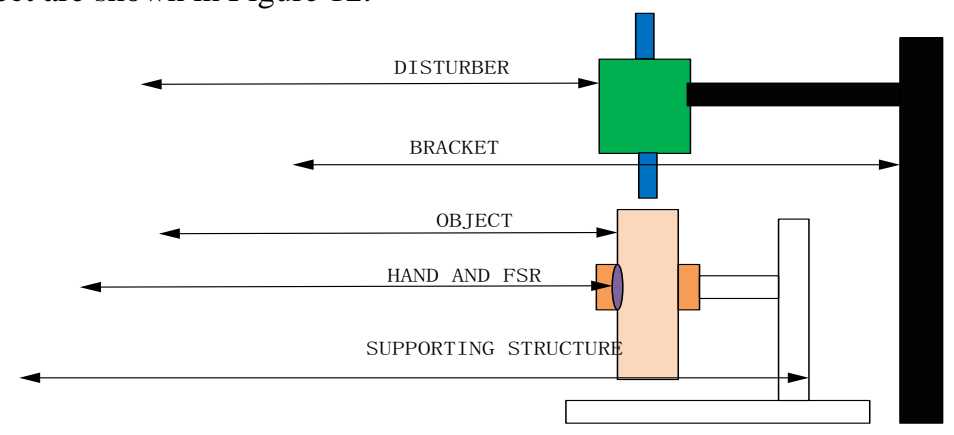

Figure 9. Disturbing device and its principles
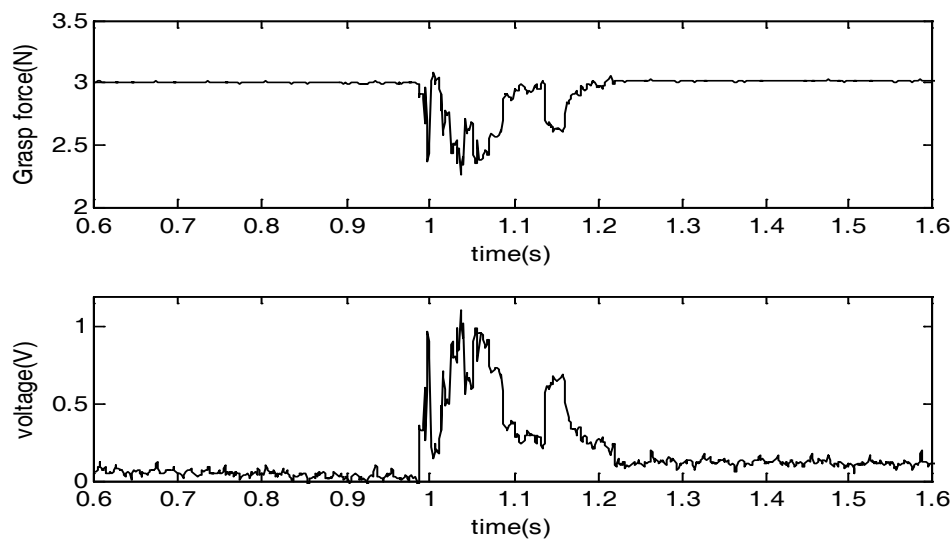

Figure 10. Force signals and electrical signals of grasping a metal cup
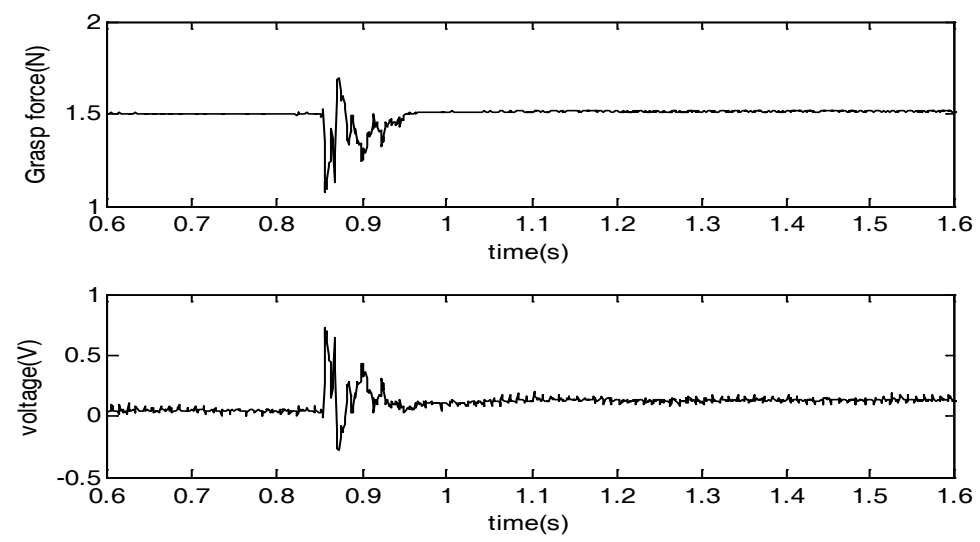

Figure 11. Force signals and electrical signals of grasping a plastic cup 

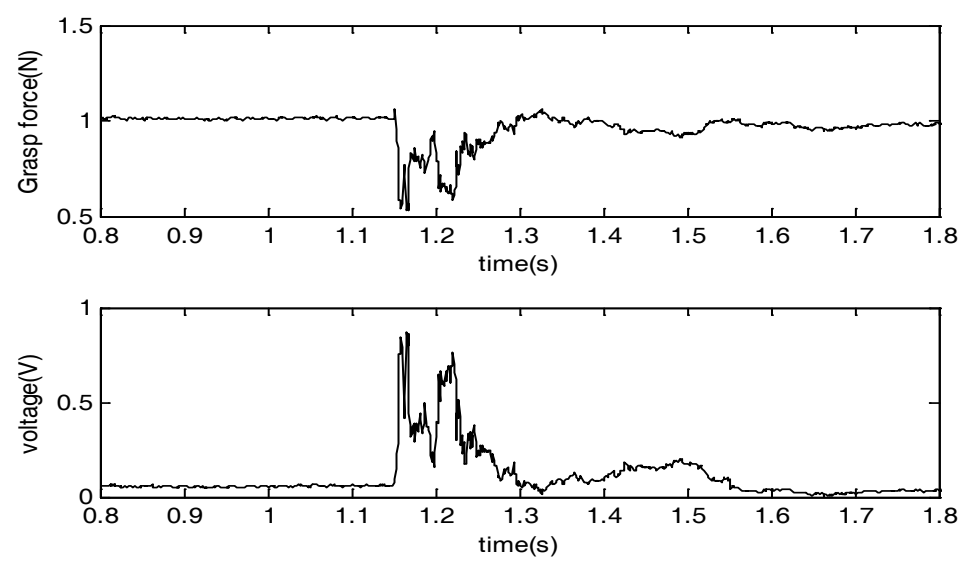

Figure 12. Force signals and electrical signals of grasping a paper cup

The weight of experimental objects, materials of objects, hitting moments, stable grasping forces, decrement of grasping force, increment of voltage and fluctuation time are described in Table 3.

Table 3. Experiment parameter of reflex control

\begin{tabular}{ccccccc}
\hline Material & Weight(g) & $\begin{array}{c}\text { Stable } \\
\text { Force(N) }\end{array}$ & $\begin{array}{c}\text { Hitting } \\
\text { Moment(s) }\end{array}$ & $\begin{array}{c}\text { Decrement } \\
\text { of force(N) }\end{array}$ & $\begin{array}{c}\text { Increment of } \\
\text { voltage(V) }\end{array}$ & $\begin{array}{c}\text { Fluctuation } \\
\text { time(s) }\end{array}$ \\
Metal & 433 & 3 & 1 & 0.7 & 0.9 & 0.25 \\
Plastic & 28 & 1.5 & 0.85 & 0.4 & 0.6 & 0.12 \\
Paper & 5 & 1 & 1.15 & 0.5 & 0.7 & 0.15 \\
\hline
\end{tabular}

Results above show that the grasp is stable before or after the disturbances happened. During the process of disturbing, the voltage applied in motors changes with the disturbing signals to make sure the prosthetic hand can grasp objects tightly without falling.

\subsection{The dual-loop control experiment}

Systematic frames of the dual-loop system are showed in Figure 13. The experimenter is a normal person whose hands are tied on a plate fixed with the prosthetic hand. When the experimenter picks up a metal cup and keeps it static, some water is added into the cup.

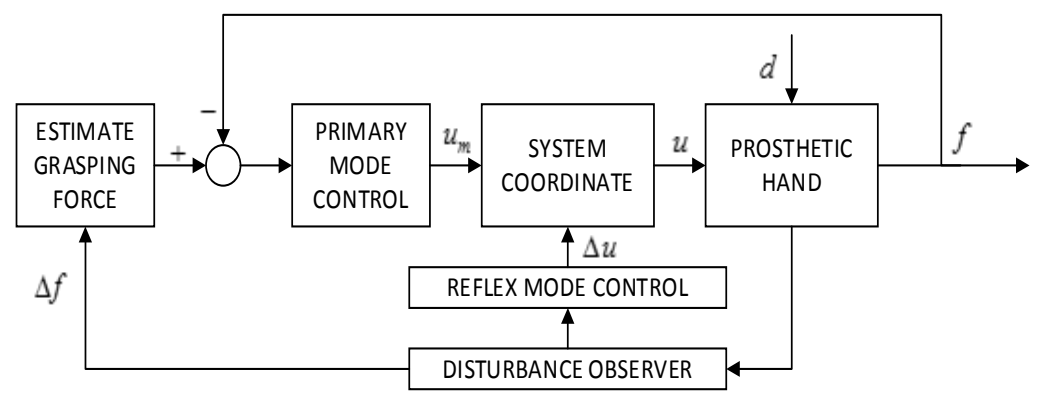

Figure 13. Systematic frames of the dual-loop system

Steps of this experiment are as follows:

(1)The prosthetic hand gets the instructions by decoding EMG signals

(2)The prosthetic hand is operated in the primary control mode

(3)The cup is picked up from the experimental platform, and disturbances occur at the time of 20s

(4)The reflex control based on the disturbance observer starts to work

(5)The prosthetic hand puts the cup on the platform and is loosen by the EMG signals 
The EMG signals are showed in Figure 14.

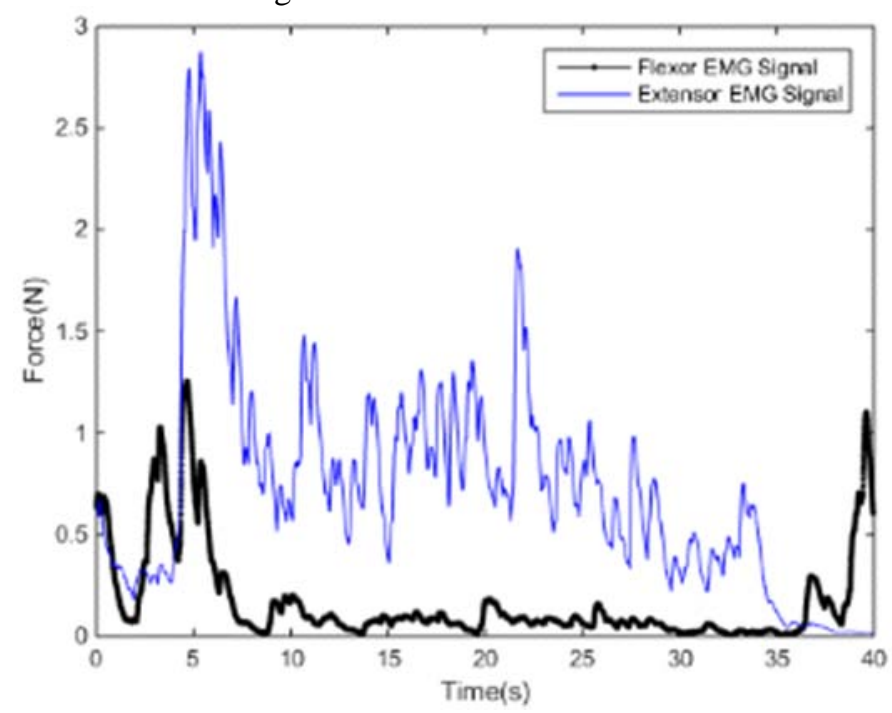

Figure 14. EMG signals of grasping a metal cup

The grasping force signals are showed in Figure 15.

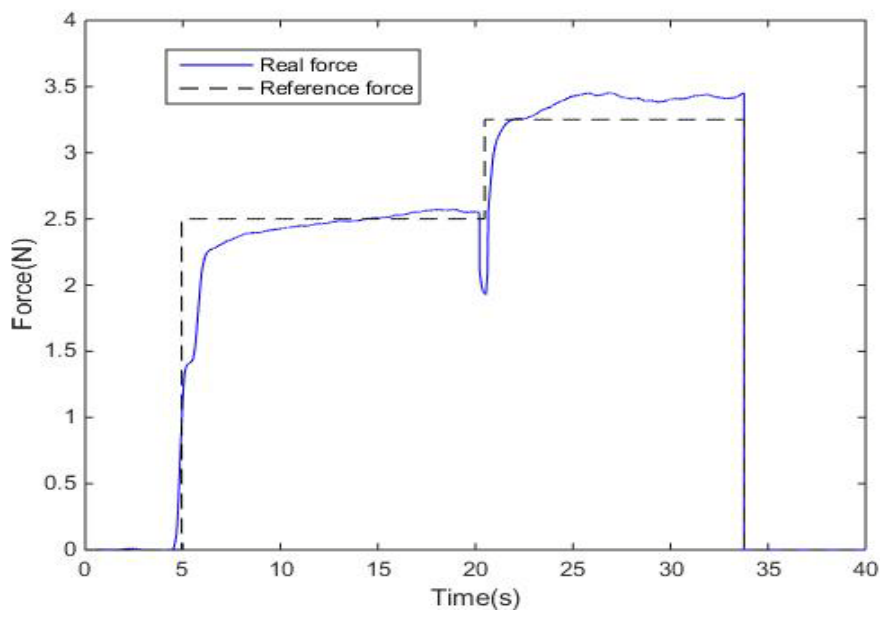

Figure 15. Force signals of grasping a metal cup

It is obvious that the primary control mode works from 0 s to $20 \mathrm{~s}$, and the error is about $0.13 \mathrm{~N}$, which is reasonable. In the $20^{\text {th }}$ seconds, water was poured into the metal cup in the experiment, and the disturbance observer monitored the prosthetic hand. Then the reflex control mode worked to increase the grasping force, making sure the object gripped by prosthetic hands did not fall. At this period, there existed overshooting of about $0.3 \mathrm{~N}$, which was acceptable. With results above, it is convinced that the dual-loop control system is efficient in controlling the grasping force.

\section{Conclusions}

In this paper, a dual-loop control system of grasping force for prosthetic hands is proposed. The primary mode of the dual-loop system aims to adjust and control the grasping force when prosthetic hands grasp different objects. The reflex mode of the dual-loop system can detect the disturbing 
signals and adjust the motor voltage to help the prosthetic hand overcome the disturbances, making sure the grasp is stable.

Experiments based on a single degree of freedom prosthetic hand were conducted. Three objects with different hardness were used in the grasping experiments, and the experimental results indicates that the dual-loop control system can make the prosthetic hand grasp smoothly without destruction and control the grasping force efficiently.

\section{Acknowledgment}

This study was supported by a grant from National Basic Research Program 973 of China (Grant Nos.2011CB013302).

\section{References}

1. T.Maeno, S.Hiromitsu, T.Kawai, "Control of Grasping Force by Estimating Stick/Slip Distribution at the Contact Interface of an Elastic Finger Having Curved Surface”, Journal of the Robotics Society of Japan, vol.19, no.1, pp.91-99,(2001).

2. A.Ikeda, Y.Kurita, J.Ueda, Y.Matsumoto, T.Ogasawara, “Grip Force Control of the Elastic Body based on Contact Surface Eccentricity During the Incipient Slip”, Journal of the Robotics Society of Japan, vol.23, no.3, pp.337-343,(2005).

3. Claudio Melchiorri, "Slip Detection and Control Using Tactile and Force Sensors”, IEEE/ASME Transactionon Mechatronis, vol.5, no.3, pp.235-243, (2000)

4. Engeberg, E.D., Meek, S.G., "Adaptive Sliding Mode Control for Prosthetic Hands to Simultaneously Prevent Slip and Minimize Deformation of Grasped Objects”, IEEE/ASME Transactions on Mechatronics 18, 376-385 (2013).

5. Cipriani, C., Zaccone, F., Micera, S. "On the Shared Control of an EMG-Controlled Prosthetic Hand”, Analysis of User-Prosthesis Interaction. IEEE Transactions on Robotics, 170-184 (2008)

6. Fougner, A., Stavdahl, Q., Kyberd, P.J., Losier, Y.G., Parker, P.A. "Control of upper limb prostheses: terminology and proportional myoelectric control - a review. IEEE Transactions on neural systems and rehabilitation engineering 20(5), 663-677 (2012).

7. Xiao-bao Deng, Xiao-gang Duan, Hua Deng, "Force Estimation for Prosthetic Hand Using Surface Electromyography”, International Conference on Test, Measurement and Computational Method, 2015. Advances in Computer Science Research, Volume 26, 99-102.

8. Yi Zhang, Xiao-gang Duan, Hua Deng, “A Method to Modify Initial Desired Force of Prosthetic Hand Based on Stiffness Estimation”, 8th International Conference on Intelligent Robotics and Applications, ICIRA 2015. Intelligent Robotics and Application Volume 9244 of the series Lecture Notes in Computer Science pp 348-358. (EI)

9. C.F. Pasluosta, H.Tims, and A.W.L. Chiu, "Slippage Sensory Feedback Nonlinear Force Control System for a Low-Cost Prosthetic Hand,” Am.J.Biomed.Sci. 2009, 1(4), 295-302.

10. Yaniger, S.I., "Force sensing resistors: A review of the technology", Electro International, 1991. IEEE,(1991).

11. Rana, N. K. "Application of force sensing resistor (FSR) in design of pressure scanning system for plantar pressure measurement," Computer and Electrical Engineering, 2009. ICCEE'09. Second International Conference on. Vol. 2. IEEE, (2009).

12. Sugeno, M., “Industrial applications of fuzzy control”, Elsevier Science Inc. (1985).

13. Zhu, D., Wu, C., "Fuzzy control of group elevators”, In: 1993 IEEE Region 10 Conference on Proceedings of the Computer, Communication, Control and Power Engineering, TENCON 1993, vol. 4, pp. 304-307 (1993).

14. Po-Rong, C., Bor-Chin, W. "Adaptive fuzzy power control for CDMA mobile radio systems”, IEEE Transactions on Vehicular Technology 45, 225-236 (1996).

15. Tang, K., Man, K.F., Chen, G., Kwong, S., “An optimal fuzzy PID controller”, IEEE Transactions on Industrial Electronics 48, 757-765 (2001). 